\title{
Probability density of nonlinear phase noise
}

\author{
Keang-Po Ho \\ StrataLight Communications, Campbell, California 95008, and Graduate Institute of Communications Engineering, \\ College of Electrical Engineering and Computer Science, National Taiwan University, Taipei, Taiwan
}

Received January 9, 2003; revised manuscript received April 25, 2003

The probability density of nonlinear phase noise, often called the Gordon-Mollenauer effect, is derived analytically. The nonlinear phase noise can be accurately modeled as the summation of a Gaussian random variable and a noncentral chi-square random variable with two degrees of freedom. Using the received intensity to correct for the phase noise, the residual nonlinear phase noise can be modeled as the summation of a Gaussian random variable and the difference of two noncentral chi-square random variables with two degrees of freedom. The residual nonlinear phase noise can be approximated by Gaussian distribution better than the nonlinear phase noise without correction. (c) 2003 Optical Society of America

OCIS codes: 190.3270, 060.5060, 060.1660, 190.4370.

\section{INTRODUCTION}

Gordon and Mollenauer ${ }^{1}$ showed that, when optical amplifiers are used to compensate for fiber loss, the interaction of amplifier noise and the fiber Kerr effect causes phase noise, often called the Gordon-Mollenauer effect or nonlinear phase noise. By broadening the signal linewidth, ${ }^{2}$ nonlinear phase noise degrades both phaseshifted keying and differential phase-shift keying systems, which have renewed attention recently. ${ }^{3-5} \mathrm{Be}-$ cause the nonlinear phase noise is correlated with the received intensity, the received intensity can be used to correct the nonlinear phase noise. ${ }^{6-8}$ The transmission distance can be doubled if the nonlinear phase noise is the dominant impairment. ${ }^{6,8}$

Usually, the performance of the system is estimated based on the variance of the nonlinear phase noise. ${ }^{1,6-8}$ The probability-density function (pdf) is necessary to better understand the system and evaluates the system performance. This paper provides an analytical expression of the pdf for the nonlinear phase noise with ${ }^{6-8}$ and without $^{1}$ the correction by the received intensity. The characteristic functions are first derived analytically, and the pdf's are the inverse Fourier transform of the characteristic functions.

\section{PROBABILITY-DENSITY FUNCTION}

For simplicity and without loss of generality, assume that the total nonlinear phase noise is ${ }^{1,6,8}$

$$
\begin{aligned}
\phi_{\mathrm{NL}}=\mid A & +\left.n_{1}\right|^{2}+\left|A+n_{1}+n_{2}\right|^{2} \\
& +\cdots+\left|A+n_{1}+\cdots+n_{N}\right|^{2},
\end{aligned}
$$

where $A$ is a real number representing the amplitude of the transmitted signal, $n_{k}=x_{k}+i y_{k}, k=1, \ldots, N$, are the optical amplifier noise introduced into the system at the $k$ th fiber span, and $n_{k}$ are independent identically distributed complex zero-mean circular Gaussian random variables with $E\left\{x_{k}^{2}\right\}=E\left\{y_{k}^{2}\right\}=E\left\{\left|n_{k}\right|^{2}\right\} / 2=\sigma^{2}$, where $\sigma^{2}$ is the noise variance per dimension per span and $E\{\}$ denotes expectation. The product of fiber nonlin- ear coefficient and the effective length per span $\gamma L_{\text {eff }}$ is ignored in Eq. (1) for simplicity. ${ }^{1,6,8}$ The random variable of Eq. (1) is a quadratic form of complex random variables ${ }^{9}$; in order to find a simplified representation, we derive its characteristic function here.

First, we consider the random variable of

$$
\begin{aligned}
\varphi_{1}=\mid A & +\left.x_{1}\right|^{2}+\left|A+x_{1}+x_{2}\right|^{2} \\
& +\cdots+\left|A+x_{1}+\cdots+x_{N}\right|^{2} .
\end{aligned}
$$

The overall nonlinear phase noise of Eq. (1) is $\phi_{\mathrm{NL}}=\varphi_{1}$ $+\varphi_{2}$, where

$$
\varphi_{2}=y_{1}^{2}+\left|y_{1}+y_{2}\right|^{2}+\cdots+\left|y_{1}+\cdots+y_{N}\right|^{2}
$$

is independent of $\varphi_{1}$ and has a pdf equal to that of $\varphi_{1}$ when $A=0$. The random variable of Eq. (2) can be expressed as

$$
\varphi_{1}=N A^{2}+2 A \mathbf{w}^{T} \mathbf{x}+\mathbf{x}^{T} \mathcal{C} \mathbf{x},
$$

where $\mathbf{w}=\{N, N-1, \ldots, 2,1\}^{T}, \mathbf{x}=\left\{x_{1}, x_{2}, \ldots, x_{N}\right\}^{T}$, and the covariance matrix $\mathcal{C}=\mathcal{M}^{T} \mathcal{M}$ with

$$
\mathcal{M}=\left[\begin{array}{ccccc}
1 & 0 & 0 & \cdots & 0 \\
1 & 1 & 0 & \cdots & 0 \\
1 & 1 & 1 & \cdots & 0 \\
\vdots & \vdots & \vdots & \ddots & \vdots \\
1 & 1 & 1 & \cdots & 1
\end{array}\right]
$$

The pdf of $\mathbf{x}$ is $\left(2 \pi \sigma^{2}\right)^{-N / 2} \exp \left(-\mathbf{x}^{T} \mathbf{x} / 2 \sigma^{2}\right)$. The characteristic function of $\varphi_{1}, \Psi_{\varphi_{1}}(\nu)=E\left\{\exp \left(j \nu \varphi_{1}\right)\right\}$, is

$$
\Psi_{\varphi_{1}}(\nu)=\frac{\exp \left(j \nu N A^{2}\right)}{\left(2 \pi \sigma^{2}\right)^{N / 2}} \int \exp \left(2 j \nu A \mathbf{w}^{T} \mathbf{x}-\mathbf{x}^{T} \Gamma \mathbf{x}\right) \mathrm{d} \mathbf{x},
$$

where $\Gamma=\mathcal{I} /\left(2 \sigma^{2}\right)-j \nu \mathcal{C}$ and $\mathcal{I}$ is an $N \times N$ identity matrix. Using the relationship of

$$
\begin{aligned}
\mathbf{x}^{T} \Gamma \mathbf{x}-2 j \nu A \mathbf{w}^{T} \mathbf{x}= & \left(\mathbf{x}-j \nu A \Gamma^{-1} \mathbf{w}\right)^{T} \Gamma\left(\mathbf{x}-j \nu A \Gamma^{-1} \mathbf{w}\right) \\
& +\nu^{2} A^{2} \mathbf{w}^{T} \Gamma^{-1} \mathbf{w}
\end{aligned}
$$


with some algebra, the characteristic function of Eq. (6) is

$$
\Psi_{\varphi_{1}}(\nu)=\frac{\exp \left(j \nu N A^{2}-\nu^{2} A^{2} \mathbf{w}^{T} \Gamma^{-1} \mathbf{w}\right)}{\left(2 \sigma^{2}\right)^{N / 2} \operatorname{det}(\Gamma)^{1 / 2}},
$$

where $\operatorname{det}[\quad]$ is the determinant of a matrix. The characteristic function of Eq. (8) is

$$
\Psi_{\varphi_{1}}(\nu)=\frac{\exp \left[j \nu N A^{2}-2 \sigma^{2} \nu^{2} A^{2} \mathbf{w}^{T}\left(\mathcal{I}-2 j \nu \sigma^{2} \mathcal{C}\right)^{-1} \mathbf{w}\right]}{\operatorname{det}\left(\mathcal{I}-2 j \nu \sigma^{2} \mathcal{C}\right)^{1 / 2}} .
$$

Substituting $A=0$ into Eq. (9), the characteristic function of $\varphi_{2}$ is $\Psi_{\varphi_{2}}(\nu)=\operatorname{det}\left[\mathcal{I}-2 j \nu \sigma^{2} \mathcal{C}\right]^{-1 / 2}$. The characteristic function of $\phi_{\mathrm{NL}}$ is $\Psi_{\phi_{\mathrm{NL}}}(\nu)=\Psi_{\varphi_{1}}(\nu) \Psi_{\varphi_{2}}(\nu)$, or

$$
\begin{aligned}
& \Psi_{\phi_{\mathrm{NL}}}(\nu) \\
& \quad=\frac{\exp \left[j \nu N A^{2}-2 \sigma^{2} \nu^{2} A^{2} \mathbf{w}^{T}\left(\mathcal{I}-2 j \nu \sigma^{2} \mathcal{C}\right)^{-1} \mathbf{w}\right]}{\operatorname{det}\left(\mathcal{I}-2 j \nu \sigma^{2} \mathcal{C}\right)} .
\end{aligned}
$$

If the covariance matrix $\mathcal{C}$ has eigenvalues and eigenvectors of $\lambda_{k}, \mathbf{v}_{k}, k=1,2, \ldots, N$, respectively, the characteristic function of Eq. (10) becomes

$$
\Psi_{\phi_{\mathrm{NL}}}(\nu)=\frac{\exp \left[j \nu N A^{2}-2 \sigma^{2} \nu^{2} A^{2} \sum_{k=1}^{N} \frac{\left(\mathbf{v}_{k}^{T} \mathbf{w}\right)^{2}}{1-2 j \nu \sigma^{2} \lambda_{k}}\right]}{\prod_{k=1}^{N}\left(1-2 j \nu \sigma^{2} \lambda_{k}\right)}
$$

and can be rewritten to

$$
\Psi_{\phi_{\mathrm{NL}}}(\nu)=\prod_{k=1}^{N} \frac{1}{1-2 j \nu \sigma^{2} \lambda_{k}} \exp \left[\frac{j A^{2}\left(\mathbf{v}_{k}^{T} \mathbf{w}\right)^{2} / \lambda_{k}}{1-2 j \nu \sigma^{2} \lambda_{k}}\right] .
$$

$$
\begin{aligned}
\frac{1}{\lambda_{k}} & \approx 2\left\{1-\cos \left[\frac{(2 k+1) \pi}{2 N}\right]\right\} \\
& =4 \sin ^{2}\left[\frac{(2 k-1) \pi}{4 N}\right], \quad k=1, \ldots, N .
\end{aligned}
$$

The values of Eq. (14) are the discrete Fourier transform of each row of the matrix $\mathcal{C}^{-1}$.

With the correction of phase noise using received intensity, ${ }^{6-8}$ the residual nonlinear phase noise is

$$
\begin{aligned}
\phi_{\mathrm{RES}}=\mid A & +\left.n_{1}\right|^{2}+\left|A+n_{1}+n_{2}\right|^{2} \\
& +\cdots+\left|A+n_{1}+\cdots+n_{N-1}\right|^{2} \\
& -\left(\alpha_{\mathrm{opt}}-1\right)\left|A+n_{1}+\cdots+n_{N}\right|^{2} .
\end{aligned}
$$

As from the appendix, $\alpha_{\text {opt }} \approx(N+1) / 2$ is the optimal scale factor to correct the nonlinear phase noise of Eq. (1) using the received intensity of $\left|A+n_{1}+\cdots+n_{N}\right|^{2}$. The random variable corresponding to $\varphi_{1}$ [Eq. (4)] becomes

$$
\left(N-\alpha_{\text {opt }}\right) A^{2}+2 A \mathbf{w}_{r}^{T} \mathbf{x}+\mathbf{x}^{T} \mathcal{C}_{r} \mathbf{x},
$$

where $\mathbf{w}_{r}=\mathbf{w}-\alpha_{\text {opt }} \times\{1,1, \ldots, 1\}^{T}$ and

$$
\mathcal{C}_{r}=(\mathcal{M}-\mathcal{L})^{T}(\mathcal{M}-\mathcal{L})-\left(\alpha_{\text {opt }}-1\right) \mathcal{L}^{T} \mathcal{L},
$$

where

$$
\mathcal{L}=\left[\begin{array}{ccccc}
0 & 0 & \cdots & 0 & 0 \\
\vdots & \vdots & \ddots & \vdots & \vdots \\
0 & 0 & \cdots & 0 & 0 \\
1 & 1 & \cdots & 1 & 1
\end{array}\right]
$$

Following the procedure from Eqs. (4) to (10), the characteristic function of $\phi_{\mathrm{RES}}$ is

$$
\Psi_{\phi_{\mathrm{RES}}}(\nu)=\frac{\exp \left[-j \nu\left(N-\alpha_{\mathrm{opt}}\right) A^{2}-2 \sigma^{2} \nu^{2} A^{2} \mathbf{w}_{r}^{T}\left(\mathcal{I}-2 j \nu \sigma^{2} \mathcal{C}_{r}\right)^{-1} \mathbf{w}_{r}\right]}{\operatorname{det}\left(\mathcal{I}-2 j \nu \sigma^{2} \mathcal{C}_{r}\right)} .
$$

From the characteristic function of Eq. (12), the random variable of $\phi_{\mathrm{NL}}$ [Eq. (1)] is the summation of $N$ independently distributed noncentral chi-square $\left(\chi^{2}\right)$ random variables with two degrees of freedom. ${ }^{10}$ The eigenvalues of the covariance matrix of $\mathcal{C}$ are all positive and multiply to unity.

Without going into detail, the matrix

$$
\mathcal{C}^{-1}=\left[\begin{array}{cccccc}
1 & -1 & 0 & \cdots & 0 & 0 \\
-1 & 2 & -1 & \cdots & 0 & 0 \\
0 & -1 & 2 & \cdots & 0 & 0 \\
\vdots & \vdots & \vdots & \ddots & \vdots & \vdots \\
0 & 0 & 0 & \cdots & -1 & 2
\end{array}\right]
$$

is approximately a Toeplitz matrix for the series of $2,-1$, $0, \ldots$ For large $N$, the eigenvalues of the covariance matrix of $\mathcal{C}$ are asymptotically equal to ${ }^{11}$
The characteristic functions of $\phi_{\text {RES }}$ in the form of eigenvalues and eigenvectors are similar to those of Eqs. (11) and (12). The characteristic functions of $\phi_{\mathrm{RES}}$ have the same expression as Eq. (12) using a new set of eigenvalues and eigenvectors of the covariance matrix $\mathcal{C}_{r}$ and the vector of $\mathbf{w}_{r}$.

Except for the first and last rows, the matrix $\mathcal{C}_{r}^{-1}$ is also approximately a Toeplitz matrix for the series of $2,-1$, $0, \ldots$ For large $N$, the eigenvalues of $\mathcal{C}_{r}$ are asymptotically equal to

$$
\frac{1}{\lambda_{k}} \approx 4 \sin ^{2}\left[\frac{(k-1.25) \pi}{2(N-1)}\right], \quad k=2, \ldots, N
$$$$
\lambda_{1} \approx-\sum_{k=2}^{N} \lambda_{k}
$$ 
Other than the largest one in absolute value, the eigenvalues of $\mathcal{C}_{r}$ are all positive. All eigenvalues of the covariance $\mathcal{C}_{r}$ sum to approximately zero and multiple to $\alpha_{\mathrm{opt}}$ $-1 \approx(N-1) / 2$.

\section{NUMERICAL RESULTS AND RANDOM VARIABLE MODELS}

The pdf's of both $\phi_{\mathrm{NL}}$ [Eq. (1)] and $\phi_{\mathrm{RES}}$ [Eq. (15)] can be calculated by taking the inverse Fourier transforms of the corresponding characteristic functions of $\Psi_{\phi_{\mathrm{NL}}}(\nu)$ [Eq. (10)] and $\Psi_{\phi_{\mathrm{RES}}}(\nu)$ [Eq. (19)], respectively. Figure 1 shows the pdf of $\phi_{\mathrm{NL}}$ [Eq. (1)] and $\phi_{\mathrm{RES}}$ [Eq. (15)]. Figure 1 is plotted for the case that the optical signal-to-noise ratio $\rho_{O}=A^{2} /\left(2 N \sigma^{2}\right)=18$, corresponding to an error probability of $10^{-9}$ if the amplifier noise is the only impairment. The number of spans is $N=32$. The $x$ axis is normalized with respect to $N A^{2}$, approximately equal to the mean nonlinear phase noise from the appendix.

Figure 1 can confirm that, using the received intensity to correct for nonlinear phase noise, the standard deviation of nonlinear phase noise can be reduced by a factor of $2 .^{6-8}$ The appendix shows that the variance of nonlinear phase noise can be reduced by a factor of $\sim 4$.

From the characteristic function of Eq. (12), the random variables of both $\phi_{\mathrm{NL}}$ and $\phi_{\mathrm{RES}}$ can be modeled as the combination of $N=32$ independently distributed noncentral $\chi^{2}$ random variables with two degrees of freedom. Some studies ${ }^{1,6,7}$ implicitly assume a Gaussian distribution by using the $Q$ factor to characterize the random variables. When many independently distributed random variables with more or less the same variance are summed (or subtracted) together, the summed random variable approaches the Gaussian distribution. For the characteristic function of Eq. (12), the Gaussian assumption is valid only if the eigenvalues $\lambda_{k}$ are more or less the same. From Eq. (14), the largest eigenvalue $\lambda_{1}$ of the covariance matrix $\mathcal{C}$ is $\sim 9$ times larger than the second largest eigenvalue $\lambda_{2}$. From Eq. (20), the two largest eigenvalues $\lambda_{1}$ and $\lambda_{2}$ of the covariance matrix $\mathcal{C}_{r}$ are $\sim 5.5$ times larger than the third largest eigenvalue $\lambda_{3}$. The approximation of Eq. (14) is accurate within $3.2 \%$ for $N=32$. The approximation of Eq. (20) is not as good as that for Eq. (14) and accurate within $10 \%$ for $N=32$.

While the Gaussian assumption for both $\phi_{\mathrm{NL}}$ and $\phi_{\mathrm{RES}}$ may not be valid, other than the noncentral $\chi^{2}$ random variables with two degrees of freedom corresponding to some large eigenvalues, the other random variables should sum to a Gaussian distribution. By modeling the summation of random variables with smaller eigenvalues as a Gaussian distribution, the nonlinear phase noise of Eq. (12) can be modeled as a summation of two or three instead of $N=32$ independently distributed random variables.

Note that the variance of the noncentral $\chi^{2}$ random variables with two degrees of freedom in Eq. (12) is $4 \sigma^{4} \lambda_{k}^{2}+4 A^{2}\left(\mathbf{v}_{k}^{T} \mathbf{w}\right)^{2} \cdot{ }^{10}$ While the above reasoning just takes into account the contribution from the eigenvalue of $\lambda_{k}$ but ignores the contribution from the eigenvector $\mathbf{v}_{k}$, numerical results show that the variance of each individual noncentral $\chi^{2}$ random variable increases with the

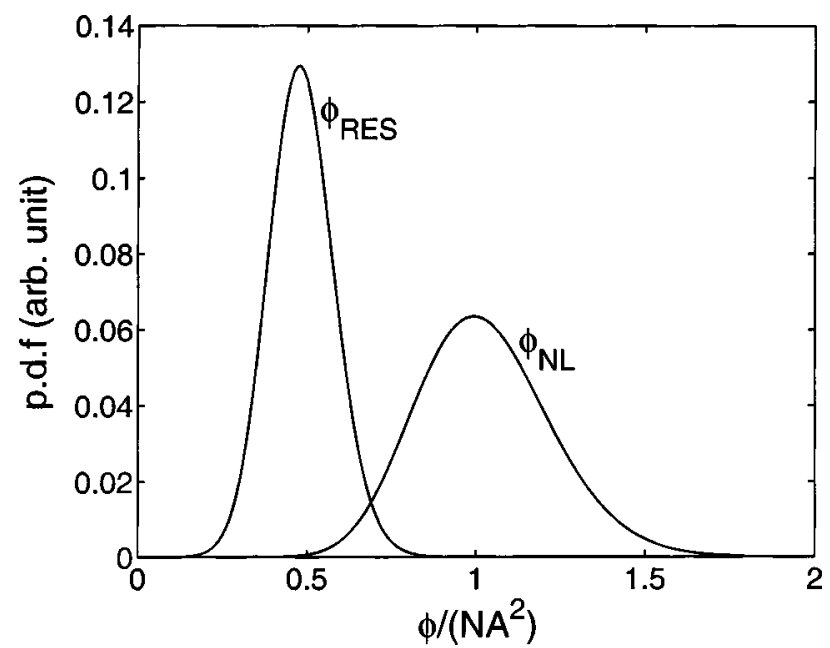

Fig. 1. pdf of both $\phi_{\mathrm{NL}}$ and $\phi_{\mathrm{RES}}$.

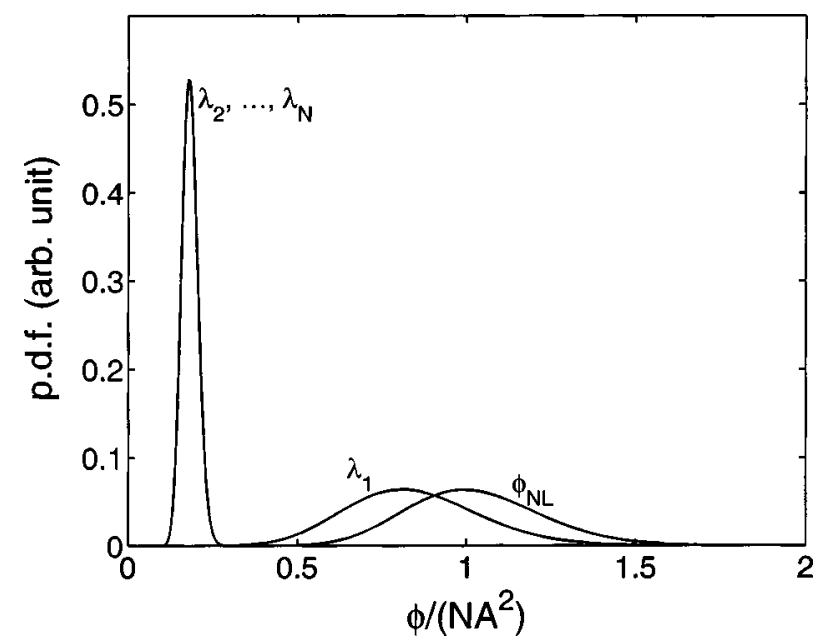

Fig. 2. pdf of $\phi_{\mathrm{NL}}$ is the convolution of a Gaussian pdf and a noncentral $\chi^{2}$ pdf with two degrees of freedom.

corresponding eigenvalue of $\lambda_{k}$. A later part of this paper also validates the argument.

From Fig. 1, the pdf of $\phi_{\mathrm{NL}}$ has significant difference from that of a Gaussian distribution. Figure 2 divides the pdf of $\phi_{\mathrm{NL}}$ into the convolution of two parts. The first part has no observable difference with a Gaussian pdf and corresponds to the second largest to the smallest eigenvalues, $\lambda_{k}, k=2, \ldots, N$, of the characteristic function of Eq. (12). The second part is a noncentral $\chi^{2}$ pdf with two degrees of freedom and corresponds to the largest eigenvalue $\lambda_{1}$, where $\sigma^{2} \lambda_{1} \approx 2 /\left(\pi^{2} \rho_{O}\right) \times N A^{2}$. The pdf of $\phi_{\mathrm{NL}}$ in Fig. 1 is also plotted in Fig. 2 for comparison. The mean and variance of the first part of the Gaussian random variable are $\sum_{k=2}^{N} A^{2}\left(\mathbf{v}_{k}^{T} \mathbf{w}\right)^{2} / \lambda_{k}+2 \sigma^{2} \lambda_{k}$ and $4 \sum_{k=2}^{N} \sigma^{4} \lambda_{k}^{2}+A^{2}\left(\mathbf{v}_{k}^{T} \mathbf{w}\right)^{2}$, respectively. The second part of the noncentral $\chi^{2}$ pdf with two degrees of freedom has a variance parameter of $\sigma^{2} \lambda_{1}$ and noncentrality parameter of $A^{2}\left(\mathbf{v}_{1}^{T} \mathbf{w}\right)^{2} / \lambda_{1} \cdot{ }^{10}$

To verify that the modeling in Fig. 2 is accurate, the cumulative tail probabilities are calculated by $\int_{-\infty}^{x} p(\xi) \mathrm{d} \xi$ and $\int_{x}^{+\infty} p(\xi) \mathrm{d} \xi$, where $p(\xi)$ is the pdf. Figure 3 shows the cumulative tail probabilities as a function of the $Q$ factor for $\phi_{\mathrm{NL}}$, defined as $Q=\left(\phi_{\mathrm{NL}}-\overline{\phi_{\mathrm{NL}}}\right) / \sigma_{\phi_{\mathrm{NL}}}$, where $\overline{\phi_{\mathrm{NL}}}$ and $\sigma_{\phi_{\mathrm{NL}}}^{2}$ are the mean and variance of the nonlinear 
phase noise given in the appendix. Using Gaussian approximation, ${ }^{1,6,7}$ this definition of the $Q$ factor gives the tail probability or error probability of $(1 / 2) \operatorname{erfc}(|Q| / \sqrt{2})$, where erfc( ) is the complementary error function. Figure 3 shows the cumulative tail probabilities calculated by numerical integration according to Eq. (10) as circles, the model as the summation of a Gaussian and a noncentral $\chi^{2}$ random variable with two degrees of freedom of Fig. 2 as solid lines, and the Gaussian assumption as dotted lines. From Fig. 3, the Gaussian approximation by the $Q$ factor is not accurate, especially for the tail probability for less than the mean. From Fig. 3, the nonlinear phase noise can be modeled very accurately as the summation of a Gaussian random variable and a noncentral $\chi^{2}$ random variable with two degrees of freedom. From Fig. 2, the noncentral $\chi^{2}$ random variable with two degrees of freedom corresponding to $\lambda_{1}$ has a very large variance, such that the pdf of $\phi_{\mathrm{NL}}$ in Fig. 1 has a significant difference with a Gaussian pdf.

Instead of the combination of $N=32$ noncentral $\chi^{2}$ random variables with two degrees of freedom, similar to the decomposition of Fig. 2, the random variable of $\phi_{\mathrm{RES}}$

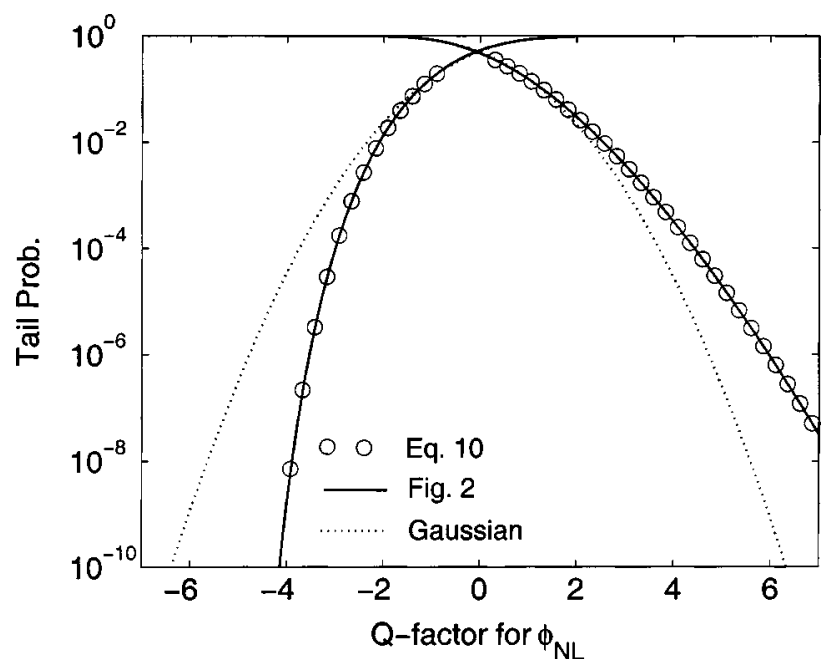

Fig. 3. Cumulative tail probability of $\phi_{\mathrm{NL}}$ as compared with the model of Fig. 2 and a Gaussian approximation.

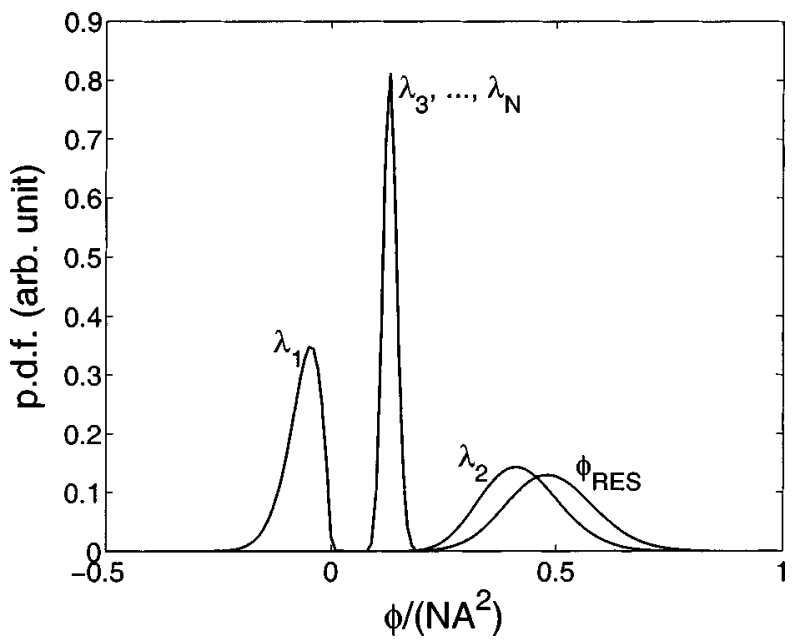

Fig. 4. pdf of $\phi_{\mathrm{RES}}$ is the convolution of a Gaussian pdf and two noncentral $\chi^{2}$ pdf's with two degrees of freedom.

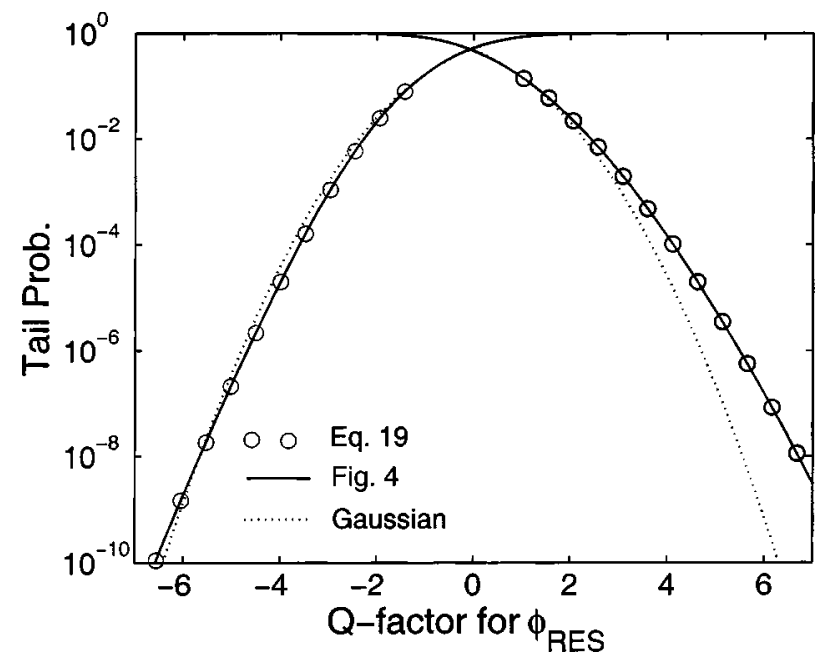

Fig. 5. Cumulative tail probability of $\phi_{\text {RES }}$ as compared with the model of Fig. 4 and a Gaussian approximation.

can be modeled as the summation of a Gaussian random variable and the difference of two noncentral $\chi^{2}$ random variables with two degrees of freedom. Figure 4 shows the pdf of $\phi_{\text {RES }}$ as the convolution of a Gaussian pdf and two noncentral $\chi^{2}$ pdf's with two degrees of freedom. The two noncentral $\chi^{2}$ random variables correspond to the two largest eigenvalues of the covariance matrix $\mathcal{C}_{r}$ with more or less the same magnitude but different signs. The Gaussian random variable corresponds to the summation of $N-2$ noncentral $\chi^{2}$ random variables with two degrees of freedom for the eigenvalues of $\lambda_{3}, \ldots, \lambda_{N}$. Because the variance parameter of $\sigma^{2} \lambda_{1}$ is negative, the corresponding random variable in Eq. (12) is the negative of a noncentral $\chi^{2}$ random variable with two degrees of freedom. The pdf corresponding to $\lambda_{1}$ in Fig. 4 is the mirror image of a noncentral $\chi^{2}$ pdf with two degrees of freedom with respect to the $y$ axis. The random variable corresponding to the combined term of both $\lambda_{1}$ and $\lambda_{2}$ in Eq. (12) is the difference of two noncentral $\chi^{2}$ random variables with two degrees of freedom.

Figure 5 shows the cumulative tail probabilities as a function of the $Q$ factor for $\phi_{\mathrm{RES}}$, defined as $Q=\left(\phi_{\mathrm{RES}}\right.$ $\left.-\overline{\phi_{\mathrm{RES}}}\right) / \sigma_{\phi_{\mathrm{RES}}}$, where $\overline{\phi_{\mathrm{RES}}}$ and $\sigma_{\phi_{\mathrm{RES}}}^{2}$ are the mean and variance of the residual nonlinear phase noise shown in the appendix. The cumulative tail probabilities calculated by numerical integration according to Eq. (19) is shown as circles, the model as the summation of a Gaussian random variable and the difference of two noncentral $\chi^{2}$ random variables with two degrees of freedom of Fig. 4 is shown as solid lines, and the Gaussian assumption ${ }^{1,6,7}$ is shown as dotted lines. From Figs. 1 and 4, the pdf of $\phi_{\text {RES }}$ resembles a Gaussian pdf with mean and variance from Ref. 8 and the appendix. The residual nonlinear phase noise of $\phi_{\text {RES }}$ can be modeled accurately as a Gaussian random variable, especially for the tail probabilities less than the mean. Even for the tail probabilities larger than the mean, the Gaussian model for $\phi_{\mathrm{RES}}$ is better than that for $\phi_{\mathrm{NL}}$. If the tail probabilities for $>10^{-5}$ is of interest, Gaussian approximation for $\phi_{\mathrm{RES}}$ can be used. 


\section{CONCLUSION}

The characteristic functions of nonlinear phase noise, with and without the correction using the received intensity, are derived analytically as a product of $N$ noncentral $\chi^{2}$ characteristic functions with two degrees of freedom. The pdf's are calculated exactly as the inverse Fourier transform of the characteristic functions. The pdf of the nonlinear phase noise can be modeled as the convolution of a Gaussian pdf and a noncentral $\chi^{2}$ pdf with two degrees of freedom. Using the received intensity to correct for the phase noise, the pdf of the residual nonlinear phase noise can be modeled accurately as the convolution of a Gaussian pdf and two noncentral $\chi^{2}$ pdf's with two degrees of freedom. The Gaussian approximation of the residual nonlinear phase noise is much better than that for nonlinear phase noise.

\section{APPENDIX A: OPTIMAL LINEAR COMPENSATOR}

This appendix shows important results from Ref. 8. The optimal scale factor to minimimize the variance of $\phi_{\mathrm{RES}}$ is

$$
\alpha_{\mathrm{opt}}=\frac{N+1}{2} \frac{A^{2}+(2 N+1) \sigma^{2} / 3}{A^{2}+N \sigma^{2}} \approx \frac{N+1}{2} .
$$

The variance of the residual nonlinear phase noise of Eq. (15) is reduced to

$$
\begin{array}{r}
\sigma_{\phi_{\mathrm{RES}}}^{2}=(N-1) N(N+1) \sigma^{2} \\
\times \frac{A^{4}+2 N \sigma^{2} A^{2}+\left(2 N^{2}+1\right) \sigma^{4} / 3}{3\left(A^{2}+N \sigma^{2}\right)}
\end{array}
$$

from that of the nonlinear phase noise of

$$
\begin{aligned}
\sigma_{\phi_{\mathrm{NL}}}^{2}= & \frac{4}{3} N(N+1) \sigma^{2}\left[\left(N+\frac{1}{2}\right) A^{2}\right. \\
& \left.+\left(N^{2}+N+1\right) \sigma^{2}\right] .
\end{aligned}
$$

The mean of the nonlinear phase noise Eq. (1) is

$$
\overline{\phi_{\mathrm{NL}}}=N\left[A^{2}+(N+1) \sigma^{2}\right] .
$$

The mean of the residual nonlinear phase noise is

$$
\overline{\phi_{\mathrm{RES}}}=\overline{\phi_{\mathrm{NL}}}-\alpha_{\mathrm{opt}}\left(A^{2}+2 N \sigma^{2}\right) \text {. }
$$

\section{REFERENCES}

1. J. P. Gordon and L. F. Mollenauer, "Phase noise in photonic communications systems using linear amplifiers," Opt. Lett. 15, 1351-1353 (1990).

2. S. Ryu, "Signal linewidth broadening due to nonlinear Kerr effect in long-haul coherent systems using cascaded optical amplifiers," J. Lightwave Technol. 10, 1450-1457 (1992).

3. A. H. Gnauck, G. Raybon, S. Chandrasekhar, J. Leuthold, C. Doerr, L. Stulz, A. Agrawal, S. Banerjee, D. Grosz, S. Hunsche, A. Kung, A. Marhelyuk, D. Maymar, M. Movassaghi, X. Liu, C. Xu, X. Wei, and D. M. Gill, "2.5 Tb/s (64 $\times 42.7 \mathrm{~Gb} / \mathrm{s})$ transmission over $40 \times 100 \mathrm{~km}$ NZDSF using RZ-DPSK format and all-Raman-amplified spans," in Optical Fiber Communication Conference (Optical Society of America, Washington, D.C., 2002), postdeadline paper FC2.

4. R. A. Griffin, R. I. Johnstone, R. G. Walker, J. Hall, S. D. Wadsworth, K. Berry, A. C. Carter, M. J. Wale, P. A. Jerram, and N. J. Parsons, " $10 \mathrm{~Gb} / \mathrm{s}$ optical differential quadrature phase shift key (DQPSK) transmission using GaAs/AlGaAs integration," in Optical Fiber Communication Conference (Optical Society of America, Washington, D.C., 2002), postdeadline paper FD6.

5. B. Zhu, L. Leng, A. H. Gnauck, M. O. Pedersen, D. Peckham, L. E. Nelson, S. Stulz, S. Kado, L. Gruner-Nielsen, R. L. Lingle, S. Knudsen, J. Leuthold, C. Doerr, S. Chandrasekhar, G. Baynham, P. Gaarde, Y. Emori, and S. Namiki, "Transmission of $3.2 \mathrm{~Tb} / \mathrm{s}(80 \times 42.7 \mathrm{~Gb} / \mathrm{s})$ over $5200 \mathrm{~km}$ of UltraWave ${ }^{\mathrm{TM}}$ fiber with $100-\mathrm{km}$ dispersionmanaged spans using RZ-DPSK format," presented of the 28th European Conference on Optical Communication, Copenhagen, Denmark, September 9-12, 2002, postdeadline paper PD4.2.

6. X. Liu, X. Wei, R. E. Slusher, and C. J. McKinstrie, "Improving transmission performance in differential phase-shiftkeyed systems by use of lumped nonlinear phase-shift compensation," Opt. Lett. 27, 1616-1618 (2002).

7. C. $\mathrm{Xu}$ and $\mathrm{X}$. Liu, "Postnonlinearity compensation with data-driven phase modulators in phase-shift keying transmission," Opt. Lett. 27, 1619-1621 (2002).

8. K.-P. Ho and J. M. Kahn, "Detection technique to mitigate Kerr effect phase noise," http://arXiv.org/physics/0211097.

9. G. L. Turin, "The characteristic function of Hermitian quadratic forms in complex normal variables," Biometrika 47, 199-201 (1960).

10. J. G. Proakis, Digital Communications, 4th ed. (McGrawHill, New York, 2000).

11. R. M. Gray, "On the asymptotic eigenvalue distribution of Toeplitz matrices," IEEE Trans. Inf. Theory IT-18, 725-730 (1972). 